\title{
Sources and Impact of Time Pressure on Opioid Management in the Safety-Net
}

\author{
Shannon Satterwhite, Kelly R. Knight, PhD, Christine Miaskowski, RN, PhD, FAAN, \\ Jamie Suki Chang, PhD, Rachel Ceasar, PhD, Kara Zamora, MA, Margot Kushel, MD
}

Purpose: This study sought to understand clinicians' and patients' experience managing chronic noncancer pain (CNCP) and opioids in safety-net primary care settings. This article explores the time requirements of safer opioid prescribing for medically and socially complex patients in the context of safety-net primary care.

Methods: We qualitatively interviewed 23 primary care clinicians and 46 of their patients with concurrent CNCP and substance use disorder (past or current). We also conducted observations of clinical interactions between the clinicians and patients. We transcribed, coded, and analyzed interview and clinical observation recordings using grounded theory methodology.

Results: Clinicians reported not having enough time to assess patients' CNCP, functional status, and risks for opioid misuse. Inadequate assessment of CNCP contributed to tension and conflicts during visits. Clinicians described pain conversations consuming a substantial portion of primary care visits despite patients' other serious health concerns. System-level constraints (eg, changing insurance policies, limited access to specialty and integrative care) added to the perceived time burden of CNCP management. Clinicians described repeated visits with little progress in patients' pain or functional status due to these barriers. Patients acknowledged clinical time constraints and reported devoting significant time to following new opioid management protocols for CNCP.

Conclusions: Time pressure was identified as a major barrier to safer opioid prescribing. Efforts, including changes to reimbursement structures, are needed to relieve time stress on primary care clinicians treating medically and socially complex patients with CNCP in safety-net settings. (J Am Board Fam Med 2019;32:375-382.)

Keywords: Chronic Disease, Chronic Pain, Grounded Theory, Minority Health, Opioids, Primary Health Care, Substance-Related Disorders, Vulnerable Populations

It is estimated that at least $11.2 \%$ of American adults experience chronic pain. ${ }^{1}$ Chronic noncancer pain $(\mathrm{CNCP})$ is a major cause of disability in

This article was externally peer reviewed.

Submitted 15 October 2018; revised 20 December 2018; accepted 6 January 2019.

From Department of Anthropology, History, and Social Medicine, University of California San Francisco, San Francisco, (SS, KRK, KZ); UCSF Medical Scientist Training Program, San Francisco (SS); School of Nursing, University of California San Francisco, San Francisco (CM); Public Health Program, Santa Clara University, Santa Clara, California (JSC); Department of Anthropology, University of California, Berkeley, Berkeley, (RC); San Francisco Veterans Affairs Medical Center, San Francisco, California, (KZ); UCSF Center for Vulnerable Populations, San Francisco General Hospital, San Francisco, California, (MK); Division of General Internal Medicine, University of California, San Francisco/San Francisco General Hospital, San Francisco, (MK). the United States. ${ }^{2}$ The co-occurrence of CNCP, substance use disorders, and other chronic conditions make treatment of CNCP challenging. Pain competes with and tends to dominate other, less symptomatic conditions. ${ }^{3,4}$ Negotiations about

Funding: Funding for this project was provided through the National Institute of Drug Abuse (NIDA; R01DA034625 and R01DA043631; Principal Investigator, Dr. Knight). In addition, Shannon Satterwhite is supported by the National Institute of General Medical Sciences (NIGMS) through the UCSF Medical Scientist Training Program (T32GM007618); a Postdoctoral Training Program in Drug Abuse Treatment/Services Research (T32DA007250) partly supported Dr. Chang; and a Career Investigator Award in Patient-Oriented Research (K24AG046372) partly supported Dr. Kushel.

Conflict of interest: none declared.

Corresponding author: Kelly Knight, PhD, University of California, San Francisco, Department of Anthropology, History and Social Medicine, 3333 California Street, San Francisco, CA, 94143 (E-mail: kelly.knight@ucsf.edu). 
opioid therapy for CNCP are shaped by the need to manage an individual's pain while reducing or avoiding the personal and community-level harms associated with opioids. ${ }^{5,6}$ These negotiations may create conflict in the clinician-patient relationship and are fraught with uncertainty about opioid efficacy and safety. ${ }^{7-9}$

While guidelines note that patients with substance use are at higher risk for opioid misuse than patients without substance use histories, patients with past or present substance use have historically been more likely to be prescribed opioids and to transition to chronic opioid therapy. ${ }^{10}$ Low-income patients with comorbid substance use and CNCP commonly receive treatment in safety-net primary care settings. Safety-net primary care settings serve low-income patients regardless of ability to pay. ${ }^{11}$ These clinics often lack resources for specialty referral, nonpharmacologic CNCP treatments, and close monitoring recommended by opioid prescription guidelines. ${ }^{5,11}$ Clinicians working in safety-net settings report a greater sense of workplace chaos and time pressure, which are associated with health care errors and clinician burnout. $^{12,13}$

Recent efforts to reform opioid prescribing practices are oriented toward the creation and implementation of standardized guidelines. ${ }^{2,14}$ Recommended practices include: limiting the duration of opioid prescriptions; consulting a statewide prescription drug monitoring database; and use of structured pain assessments, functionality assessments, pain agreements, and urine toxicology screening. ${ }^{2,14}$

A handful of studies and several professional commentaries cite time as a barrier to achieving guideline-concordant opioid prescribing in primary care. ${ }^{7,15-18}$ Time scarcity in primary care is widely acknowledged, yet its influence on management of CNCP and opioids is understudied in the current literature. None of these studies, for instance, have elaborated the sources and implications of time pressure for patients and clinicians who are trying to manage CNCP, substance use, and other comorbid chronic conditions. This article addresses this gap using qualitative methods to evaluate the experience of time pressures related to CNCP and opioid management in the safety net. Drawing on direct clinical observations and in-depth interviews, we describe the temporal experiences of safety-net primary care clinicians and their patients with co-
Table 1. Patient Participant Characteristics

\begin{tabular}{lc}
\hline Characteristic & $\mathrm{N}(\%)$ \\
\hline Age (years) & \\
$55+$ & $21(46)$ \\
40 to 54 & $18(39)$ \\
25 to 39 & $5(11)$ \\
Not recorded & $2(4)$ \\
Sex & \\
Female & $25(54)$ \\
Male & $21(46)$ \\
Race/ethnicity & \\
African American & $28(61)$ \\
White & $14(30)$ \\
Latino & $3(7)$ \\
Not recorded & $1(2)$ \\
Substance use (past or present) & \\
Cocaine & $30(65)$ \\
Alcohol & $26(57)$ \\
Marijuana & $21(46)$ \\
Methamphetamine & $14(30)$ \\
Heroin & $11(24)$ \\
Nonprescribed opioids & $3(7)$ \\
\hline
\end{tabular}

*Patients may have reported use of more than one substance

morbid substance use and CNCP. We elucidate contextual factors that contribute to the perception and experience of time scarcity and its effects on quality of CNCP management.

\section{Methods}

\section{Recruitment and Sample Selection}

We recruited and interviewed 23 primary care clinicians from 6 urban and suburban safety-net primary care clinics across 4 counties in the San Francisco Bay Area by using a purposeful sampling approach. Purposive sampling in qualitative research is the identification and selection of information-rich cases to answer study questions. ${ }^{19}$ Four of the 6 sites were family health centers. We defined a clinician as a physician, nurse practitioner, or physician assistant who provides longitudinal primary care. In California, nurse practitioners and physician assistants can prescribe schedule II, III, $\mathrm{IV}$, and V controlled substances, including opioids.

For each participating clinician, we recruited between 1 and 4 of their patients who had both CNCP and a history of past or current substance use (see Table 1). Potential patient participants were identified by their primary care provider based on these criteria. We assessed patients' de- 
mographics, prescribed pain medications, and substance use with a questionnaire that was developed by the researchers. Clinicians and patients received a $\$ 50$ gift card for their participation. One clinic did not allow clinicians to accept compensation. All participants provided written informed consent. This study was approved by the institutional review board.

The research team included an attending internist, a professor of nursing with expertise in pain management, and medical anthropologists and sociologists. The clinicians provided guidance and expertise in the development of the interview guide and contributed to the data analysis. Data collection was conducted by nonclinician study staff with training and experience in qualitative research.

\section{Data Collection}

Study researchers conducted semistructured, openended interviews with clinicians about their experiences caring for patients with CNCP and substance use, their perceptions of the risks of opioids, and their experiences with the implementation of opioid monitoring policies. We conducted semistructured, open-ended interviews with patients about their experiences with CNCP and substance use, their perceptions of the risks of prescription opioids and illicit drugs, experiences with opioid monitoring policies, and their impressions of their clinicians' views on opioid therapy. We recorded interviews and transcribed them verbatim. We continued recruitment and interviews until we reached thematic saturation, meaning that no new themes emerged from ongoing data analysis. ${ }^{19}$

In addition, we observed and audio-recorded 26 clinical encounters involving 19 dyads of participating clinicians and patients. Observations occurred during the next appointment between each dyad after we had interviewed each clinician and patient separately. The observer did not interact with the dyad during the visit. The audio recordings were transcribed verbatim.

\section{Data Analysis}

We used a modified grounded theory approach to code and analyze interview and clinical observation transcripts using ATLAS.ti. ${ }^{20,21} \mathrm{We}$ combined deductive codes, based on broad topics from the semistructured interview guide, with inductive codes (ie, those that emerged from the data, analytic discus- sions, and memos). After successive iterations of independently coding and discussing transcripts, we developed distinct codebooks for patients' and clinicians' interviews and clinical observations. Two researchers coded each transcript. We resolved coding discrepancies through consensus. For this analysis, SS reviewed all interview and clinical observation transcripts and reviewed coded data related to clinics' and patients' resources, continuity, and medical complexity. The research team discussed emergent themes.

\section{Results}

Time pressure was a major barrier to effective management of CNCP. Related themes that emerged included: CNCP management as a drain on clinicians' time and emotional energy (see Table 2), pain and opioids drawing time and attention away from other patients and health concerns, insurance requirements (eg, narrow and changing formularies, prior authorization for medications), short visit times and lack of continuity, and the effects of time pressure and opioid prescribing policies on patients. The influence of time pressure was so strong that one clinician stated: "I think maybe the overprescribing [of opioids] is a reflection that we have no time with people."

\section{Pain as a Time Drain: Clinicians' Perceptions}

Many clinicians described CNCP management, and particularly opioid prescribing and monitoring, as time-intensive and stress-inducing in the context of short visits for complex patients (Table 2). Many noted the tendency for patient-clinician interactions to feel like a "struggle," fraught with conflict. Clinicians felt that CNCP patients were "tak[ing] time away from other patients," as well as from the same patients' other medical needs. Inadequate functional assessment initially set clinicians up for ongoing conflict with patients.

One clinician observed that clinicians' perceptions of time spent on opioid management reflected the emotional intensity of CNCP management. She and her colleagues perceived that CNCP patients on chronic opioid therapy demanded a significant amount of mental and emotional energy. She identified intensity of suffering and lack of improvement as factors that contributed to this perception. 
Table 2. Clinicians' Perspectives on Pain and Opioid Management

\section{Pain as Time Drain: Clinician Perspectives}

Theme: Pain management takes time from other patients and health concerns

"My pain management patients drain my time. I don't want to say 'drain' but it's true, at least that's how I feel. My perception is that they drain my time and that puts me behind in the rest of my schedule, because there's always a struggle... And that takes away from the other patients that I need to spend more time with."

"From a patient's perspective, pain and mental health and well-being are the most, are frequently the most pressing issues, and understandably so. But there also are other big things that could be going on with people from a health perspective that are important, too, and it all needs to fit into the time. That's hard."

Theme: Pain and opioid management are often fraught with conflict

"[In] terms of trajectory with patients. . . either if I'm inheriting them or if I'm starting over with them, or even starting with these new patients, [I try] to establish some functional goals. . I totally do not have time to do any of [it] but it's not an option [not to]. If you don't do it the whole relationship ends up being a disaster. . . because every time you see them you're just arguing about whether or not it's [the pain's] better, whether it [opioid medication] makes them better or worse. .."

Theme: The intensity and chronicity of patient suffering weighs on providers

"[If] you ask anybody in [this clinic] what percentage of our patients are on chronic opioids, they'll tell you some completely inflated number. . I think [it's] because [of] the psychologic[al] space that these [CNCP] patients take up in people's minds. And that's because nobody's suffering more than a person with chronic pain. . . so you develop these really passionate and intense relationships with people because. . . they're trusting you with their suffering and you want to help them. And sometimes we help people and they move in a positive direction and sometimes we

don't. . . [The] other thing that would happen is. . you're trying to get them healthier and they'd no-show to all your visits and they would always come to. . . their refill appointment."

CNSP, chronic noncancer pain.

Furthermore, this clinician referenced "refill appointments." In the county health systems within which the study took place, prescriptions for opioids schedule II, III, and IV require a signed paper prescription that cannot be transmitted by fax. ${ }^{22}$ Schedule II medications can only be prescribed in 30-day increments without refills. Refill appointments refer to monthly visits to the clinic, separate from a regular primary care appointment, during which patients pick up a secure prescription to take to the pharmacy. Some patients' tendency to miss standard primary care visits but then consistently pick up refills was frustrating and discouraging for clinicians.
Table 3. Contextual Factors Identified by Clinicians

Insurance, Inherited Patients, and the 15-Minute Visit: Contextual Factors

Theme: Reimbursement structures limit time for pain management in the safety-net

"I went to that [CME and] they [said], "You got to spend at least an hour [in] the first visit with each [CNCP] patient," and all these internists were saying, "Okay, yeah, no problem." And they're going to bill the patient for the time. I'm working in a Medi-Cal [Medicaid] clinic, I can't do that. So, I went, "Yeah, in an ideal world." So, I heard all this stuff that I'm supposed to be doing, taking a complete history, complete addiction history. . . But I don't have time to do what I am supposed to do in terms of proper treatment, opioid treatment, so I cut corners a bit."

Theme: Thorough initial assessment is difficult in brief visits, but essential for future care

"[Any] time I get a new patient who has pain it's like the first visit. . . as soon as I can get to the place where everything else feels stable enough for me to talk about the pain, that's all I'll do. Because I think that initial pain assessment is so important and often times lost to me when you inherit [another clinician's patient]... You need time to listen. . . it takes more than fifteen minutes often. . I just see that as such an important initial step in coming up with good plans and setting up people for expectations."

Theme: Visit duration and availability pose challenges to pain management

"[A] new patient for us should be a 30-minute visit but there's so many new patients sometimes that new patients will be put into a 15 -minute visit. And even 30 minutes is not enough for a lot of complex issues... And, yes, we do often have a culture where we deal with one or two problems on one issue because we don't have the time and we'll bring them back [for a follow-up visit]."

"It's very hard to get an appointment in the clinic [when] you want one, and so one defense mechanism that almost all the doctors there use is to see people more frequently than they actually need to because everyone's afraid of their patients falling through the cracks and that just makes the problem [of clinical availability] worse."

CNSP, chronic noncancer pain.

\section{Insurance, Inberited Patients, and the 15-Minute Visit: Contextual Factors}

Clinicians emphasized that the structure of the safety-net health delivery system contributed to the challenges they faced in treating patients with CNCP (Table 3). For example, several providers cited narrow and changing formularies and lack of insurance coverage for nonpharmacologic treatments as barriers to treating patients with CNCP. One of the most salient contextual factors was the short duration of visits.

One clinician reported that a continuing medical education session alerted him to how limitations in the safety-net reimbursement structure placed constraints on the "proper treatment" of CNCP. This clinician felt like it was not the absence of adequate 
assessment tools that kept him from providing good care but lack of reimbursement for clinical time he would need to use them.

Other clinicians also emphasized the challenges of conducting CNCP assessments and treatment planning during short clinical visits. For example, another clinician described quickly working through a patient's other medical conditions to try to create enough time to listen to them describe their pain. She felt this time was particularly important early on to set the course for treatment. Clinicians reported that these initial conversations were high stakes because they affected the course of patientclinician relationships and future clinical encounters.

Several clinicians mentioned "inheriting" other clinicians' patients (Tables 2 and 3). Although many clinicians, in line with current guidelines, avoided initiating opioids for CNCP patients, clinicians were seeing patients on chronic opioids who were new to them. ${ }^{23}$ In short visits, they struggled to orient themselves to the history behind patients' current opioid regimens and to establish the rapport necessary to discuss opioid tapering and alternative pain management strategies. Lack of continuity was a significant barrier to optimal CNCP management.

These clinicians' comments highlight that pain and opioids are separate but related issues. It was difficult for patients and clinicians to address concerns about multiple health, pain, and opioid-related concerns in a single visit. Insurance issues and difficulty accessing specialists and other services consumed a large portion of some patient encounters.

Responding to the medical and social complexity of their patients, one solution that clinicians had for the time shortage was to schedule frequent follow-up visits. This scheduling practice, in turn, created challenges in terms of patients' access to care. Some clinicians scheduled frequent visits with socially vulnerable patients to bolster continuity, concerned that a lack of appointments available on short notice would prevent patients from accessing care when needed. As described by one clinician in Table 3, this scheduling strategy exacerbated limited appointment availability.

\section{"I am on a time limit with you": Patients' Awareness of Time Challenges}

Some patients recognized and responded to the time burden on clinicians. For example, one patient teased her clinician for interjecting several times as she presented her concerns:

Patient: Right, he said it was like-

Clinician: Tendinitis, tendinosis?

Patient: Something like that in the, in the foot, he said that.

Clinician: Okay. Which foot did he work on, both of them?

Patient: No, that is why, we fixin' to get to that point.

Clinician: Okay, I will let your roll out your story.

Patient: You know... because I am on a time limit with you.

Clinician: That is okay, you are the last one.

This patient had an appointment toward the end of the clinic session. Her clinician did not refute the idea of a time limit. Rather, she said that she could be more flexible because no additional patients were waiting.

Another patient contrasted her clinician to other clinicians who act "like drug dealers," prescribing without careful discussion nor assessment (Table 4). At the same time, she was aware that her clinician was overwhelmed. Another patient with a complex medical history, including major surgery, spoke of the lack of continuity she experienced and how her health improved once she had one steady primary care clinician. Patients were critical of the effects of short visits and lack of continuity on their care.

\section{Time Burdens for Patients with CNCP}

Many patients' lives were structured around frequent medical and other appointments. When members of the study team scheduled and conducted clinic observations, we learned about the patients' many appointments. In one case, because of the way secure prescriptions were managed in his clinic, a patient had separate appointments on the same day, 1 for a regular visit with his primary care clinician and another to pick up his prescription. Frequent visits, combined with requirements to come in person to pick up signed paper opioid prescriptions, were burdensome for many patients.

Several patients argued that clinicians should be able to distinguish those individuals who are misusing medications from others. One patient, for example, explained why patients like him should not be burdened with extra trips to the clinic to pick up their prescriptions (Table 4). As discussed above, clinicians in this setting can no longer "call 
Table 4. Patients' Perspectives on Pain and Opioid Management

Patient Perspectives

Theme: Patients sense that providers are rushed

"Some providers actually care about what's wrong with a person. My new provider is like... I think he's overwhelmed with how many patients he has, but at the same time he still tries to listen to you. [He] doesn't just go, 'Here you go, here's your [opioid], go, bye.' You know, he does pay attention but I think he's really overwhelmed. . you can just see it in his face."

Theme: Continuity is important for pain and opioid management

"Every time I came to the clinic, it was always a new doctor. So, I would always have to explain my story all over again to a new doctor. ... [My current clinician] said that she would take me. And so ever since then [my clinician] got me off of all my medicine... and I'm actually getting

[better]... But with [the other doctors] I never got medicine [opioids], I got medicine twice and they're like, 'Here, just take a couple,' and that would be it. But for me it wasn't just, 'Give me medicine to make the pain go away.' I want help to make sure if there's something that they can do to make the pain go away without medicine. That's what me and [my clinician] are trying to figure out right now."

Theme: Patients feel stigmatized and burdened by timeintensive monitoring requirements

"[If] the doctor know [sic] my background and I have chronic pains. . . I feel like [the clinicians] should call my meds in, I shouldn't have to come up there and get a prescription... I feel like that because it don't make no sense. It's basically you're going in there without an appointment but it seems like it is appointment because you have to sit in there and you have to actually wait to get it and you're not going in the back [to a clinic room]. .. So, you won't be able to say nothing to that doctor or anything, [and] you come back two weeks later to come to the doctor. I feel like they need to better up on, on serious people that really, really have chronic pain."

in" or electronically prescribe opioids. Prescriptions have to be written by hand on secure prescription pads and hand-delivered to the pharmacy. Clinicians and patients alike are constrained by policy intended to manage risks. In some cases, patients are required to pick up a new prescription each month and may not see their clinician during this "refill appointment." This patient felt it was unjust to patients with pain to have to make separate visits to the clinic and wait to pick up their prescriptions. Patients reported feeling that increased restrictions in opioid prescribing were a response to the behaviors of other patients who misused, were unfairly applied to them, ${ }^{24}$ and created additional time burdens such as refill appointments.

\section{Discussion}

Insufficient time to provide necessary services is a widespread concern in primary care. ${ }^{25-27}$ Findings from this study suggest that the time required for appropriate assessment of CNCP far exceeds the time available in safety-net primary care settings. The use of opioids to manage CNCP when access to alternative treatments is limited by insurance coverage and long waitlists, contributes to this perception among safety-net clinicians. ${ }^{5}$ Several of the current strategies for managing opioid risks (eg, prescription drug monitoring programs, pain agreements, functional status assessments, and urine toxicology) require significant time investment, which exacerbates time pressure in this setting. Time pressure can escalate tensions between patients and clinicians and compromise the quality of care for vulnerable patients. ${ }^{28}$ The observation by one of the providers in this study, that "overprescribing [of opioids] is a reflection that we have no time with people," merits further investigation. It suggests that although it is well known that time is a challenge in primary care generally, the specific implications of time pressure for patterns of opioid prescribing and discontinuation should be examined as prescribing norms shift. ${ }^{29}$

Research has shown that recommended practices can support clinicians in their efforts to manage opioid-related risks. ${ }^{23,30}$ Clinicians felt that new strategies were needed to increase the time available for CNCP and opioid management and improve the quality of care for medically complex patients with $\mathrm{CNCP}$ and substance use who are already receiving opioids. Alternative payment models, such as bundled payments and capitation, are becoming more common and could support the reallocation of physician time and the incorporation of more interdisciplinary care in safety-net clinical settings. ${ }^{25,31-34}$ At the same time, low payment rates and the mix of payers and incentives leave patient visit volume a primary driver of financial sustainability in safety-net primary care. ${ }^{32,35}$ Furthermore, research has warned of the unintended consequences of pay-for-performance for safety-net systems. ${ }^{13,36,37}$ Alternative reimbursement structures that support safety-net primary care should be explored to allow more time and continuity of care for patients with CNCP.

Many emerging opioid management policies and procedures increase the time and organization required for patients to obtain pain medications while structural barriers (eg, insurance limitations, access to referrals, and transportation) limit access to nonpharmacological treatments. Concerns over 
opioid safety must be balanced with the burden, including time demands, that regulatory practices place on patients. Such burdens may unintentionally undermine the goal of increasing patient function and well-being. ${ }^{38-40}$

This study has several limitations. Due to the qualitative nature of the study, the sample size was small, limiting generalizability. We focused on clinicians in a primary care provider role, excluding other individuals who play an important role in CNCP management (eg, medical assistants, nurses, pharmacists, and social workers). We did not use standard measures of patient or clinician experience and we did not measure time use but relied on participants' reports to better understand their perceptions.

\section{Conclusion}

Time pressure is a significant obstacle to effective management of CNCP in safety-net primary care clinics. Current efforts to develop tools and policies that facilitate the assessment and treatment of pain and management of opioids need to be tailored to this setting. Payment models that reimburse for the time necessary for CNCP assessment would help to alleviate time pressure as a barrier to adequate and appropriate pain management. As changes are implemented, ${ }^{29}$ caution is needed in addressing the ways in which the time demands of new policies impact care for socially vulnerable, medically complex patients in safety-net settings.

The authors thank the clinicians and patients who participated in this study.

To see this article online, please go to: bttp://jabfm.org/content/ 32/3/375.full.

\section{References}

1. Nahin RL. Estimates of pain prevalence and severity in adults: United States, 2012. J Pain 2015; 16:769-80.

2. Chou R, Fanciullo GJ, Fine PG, et al. Clinical guidelines for the use of chronic opioid therapy in chronic noncancer pain. J Pain 2009;10:113-30.

3. Butchart A, Kerr EA, Heisler M, Piette JD, Krein SL. Experience and management of chronic pain among patients with other complex chronic conditions. Clin J Pain 2009;25:293-8.

4. Krein SL, Hofer TP, Holleman R, Piette JD, Klamerus ML, Kerr EA. More than a pain in the neck: how discussing chronic pain affects hypertension medication intensification. J Gen Internal Med 2009; 24:911-6.
5. Cristobal K. Striking a balance: safety-net leaders explore solutions to the prescription painkiller epidemic. Oakland (CA): California HealthCare Foundation; 2015.

6. Ballantyne JC, Mao J. Opioid therapy for chronic pain. N Engl J Med 2003;349:1943-53.

7. Harle CA, Bauer SE, Hoang HQ, Cook RL, Hurley RW, Fillingim RB. Decision support for chronic pain care: how do primary care physicians decide when to prescribe opioids? A qualitative study. BMC Fam Pract 16:48, 2015.

8. Merrill JO, Rhodes LA, Deyo RA, Marlatt GA, Bradley KA. Mutual mistrust in the medical care of drug users: the keys to the "narc" cabinet. J Gen Internal Med 2002;17:327-33.

9. Matthias MS, Krebs EE, Collins LA, Bergman AA, Coffing J, Bair MJ. "I'm Not Abusing or Anything": Patient-physician communication about opioid treatment in chronic pain. Patient Educ Couns 2013;93: 197-202.

10. Morasco BJ, Gritzner S, Lewis L, Oldham R, Turk DC, Dobscha SK. Systematic review of prevalence, correlates, and treatment outcomes for chronic noncancer pain in patients with comorbid substance use disorder. Pain 2011;152:488-97.

11. Institute of Medicine. America's safety net: intact but endangered. Available from: http://www.nationalacademies.org/hmd/Reports/2000/Americas-HealthCare-Safety-Net-Intact-but-Endangered.aspx. Published 2000. Accessed October 18, 2015.

12. Perez HR, Beyrouty M, Bennett K, et al. Chaos in the clinic: characteristics and consequences of practices perceived as chaotic. J Healthc Qual 2017;39: 43-53.

13. Varkey AB, Manwell LB, Williams ES, et al. Separate and unequal: clinics where minority and nonminority patients receive primary care. Arch Intern Med 2009;169:243-50.

14. Dowell D, Haegerich TM, Chou R. CDC Guideline for prescribing opioids for chronic pain-United States, 2016. JAMA 2016;315:1624-45.

15. Barry DT, Irwin KS, Jones ES, et al. Opioids, chronic pain, and addiction in primary care. J Pain 2010;11:1442-50.

16. Kirschner N, Ginsburg J, Sulmasy LS. Prescription drug abuse: executive summary of a policy position paper from the American College of Physicians. Ann Internal Med 2014;160:198-200.

17. Krebs EE, Bergman AA, Coffing JM, Campbell SR, Frankel RM, Matthias MS. Barriers to guidelineconcordant opioid management in primary care-a qualitative study. J Pain 2014;15:1148-55.

18. Reuben DB, Alvanzo AA, Ashikaga T, et al. National Institutes of Health Pathways to Prevention Workshop: the role of opioids in the treatment of chronic pain. Ann Internal Med 2015;162:295-300. 
19. Patton M. Qualitative research and evaluation methods. 3rd ed. Thousand Oaks (CA): Sage Publications; 2002.

20. Strauss A, Corbin JM. Basics of qualitative research: techniques and procedures for developing grounded theory. 4th ed. Thousand Oaks (CA): SAGE Publications; 2014.

21. Atlas.ti. ATLAS.ti Scientific Software Development $\mathrm{GmbH}$. Available from: www.atlasti.com.

22. Board of Pharmacy Regulations. 2017 Lawbook for Pharmacy. Sacramento (CA): California State Board of Pharmacy, 2017.

23. Katz MH. Opioid prescribing for chronic pain: not for the faint of heart. 7 AMA Intern Med. 2016;176: 599-601.

24. Hurstak E, Kushel M, Miaskowski C, et al. The risks of opioid treatment: perspectives of primary care practitioners and patients from safety-net clinics. Subst Abus 2017;38:213-21.

25. Osborn R, Moulds D, Schneider EC, Doty MM, Squires D, Sarnak DO. Primary care physicians in ten countries report challenges caring for patients with complex health needs. Health Aff 2015;34: 2104-12.

26. Sinsky CA, Willard-Grace R, Schutzbank AM, Sinsky TA, Margolius D, Bodenheimer T. In search of joy in practice: a report of 23 high-functioning primary care practices. Ann Fam Med 2013;11:272-8.

27. Østbye T, Yarnall KSH, Krause KM, Pollak KI, Gradison M, Michener JL. Is there time for management of patients with chronic diseases in primary care? Ann Fam Med 2005;3:209-14.

28. Higashi RT, Tillack A, Steinman MA, Johnston CB, Harper GM. The 'worthy' patient: rethinking the 'hidden curriculum' in medical education. Anthropol Med 2013;20:13-23.

29. Knight KR, Kushel M, Chang J, Ceasar R, Zamora $\mathrm{K}$, Miaskowski C. Opioid pharmacovigilance: primary care providers describe the social history and unintended consequences of opioid prescribing for patients with co-occurring chronic non-cancer pain and substance use. Soc Sci Med 2017;186:87-95.
30. Ceasar R, Chang J, Zamora K, et al. Primary care providers' experiences with urine toxicology tests to manage prescription opioid misuse and substance use among chronic noncancer pain patients in safety net health care settings. Subst Abus 2016;37:154-60.

31. Basu S, Phillips RS, Song Z, Bitton A, Landon BE. High Levels Of capitation payments needed to shift primary care toward proactive team and nonvisit care. Health Aff 2017;36:1599-605.

32. Friedberg MW, Chen PG, White C, et al. Effects of health care models on physician practice in the United States. Rand Health Q 2015;5:8.

33. Ku L, Frogner BK, Steinmetz E, Pittman P. Community health centers employ diverse staffing patterns, which can provide productivity lessons for medical practices. Health Aff 2015;34:95-103.

34. Purnell TS, Calhoun EA, Golden SH, et al. Achieving health equity: closing the gaps in health care disparities, interventions, and research. Health Aff 2016;35:1410-5.

35. Biener AI, Selden TM. Public and private payments for physician office visits. Health Aff 2017;36: $2160-4$.

36. Friedberg MW, Reid RO, Timbie JW, et al. Federally qualified health center clinicians and staff increasingly dissatisfied with workplace conditions. Health Aff 2017;36:1469-75.

37. Lewis VA, Fraze T, Fisher ES, Shortell SM, Colla $\mathrm{CH}$. ACOs serving high proportions of racial and ethnic minorities lag in quality performance. Health Aff 2017;36:57-66.

38. Cooper A. Time seizures and the self: institutional temporalities and self-preservation among homeless women. Cult Med Psych 2015;39:162-85.

39. Foucault M. Discipline and punish: the birth of the prison. 1995 ed. New York (NY): Vintage Books; 1995.

40. Rhodes LA. Emptying beds: the work of an emergency psychiatric unit. Berkeley (CA): University of California Press; 1991. 